PROCEEDINGS OF THE

AMERICAN MATHEMATICAL SOCIETY

Volume 127, Number 6, Pages 1671-1682

S 0002-9939(99)04660-2

Article electronically published on February 4, 1999

\title{
NORM OF CONVOLUTION BY OPERATOR-VALUED FUNCTIONS ON FREE GROUPS
}

\author{
ARTUR BUCHHOLZ
}

(Communicated by Palle E. T. Jorgensen)

\begin{abstract}
We present a connection between the Leinert sets and the noncrossing two-partitions and we use this connection to give a simple proof of the free Khintchine inequality in discrete non-commutative $L_{p}$-spaces. Moreover we extend the inequality of Haagerup-Pisier,
\end{abstract}

$$
\left\|\sum_{g \in S} \lambda(g) \otimes a_{g}\right\|_{C_{\lambda}^{*}\left(F_{n}\right) \otimes A} \leq 2 \max \left\{\left\|\sum_{g \in S} a_{g}^{*} a_{g}\right\|^{\frac{1}{2}},\left\|\sum_{g \in S} a_{g} a_{g}^{*}\right\|^{\frac{1}{2}}\right\},
$$

where $\lambda$ is the left regular representation of the group $F_{n}, a_{g}$ are elements of the $C^{*}$-algebra $A$, and $S$ is the set of the words with length one, to the set $S$ of the words with arbitrary fixed length.

\section{INTRODUCTION}

In this paper we treat two inequalities (scalar and operator) related to norms of convolution operators on free groups.

Section 1 is devoted to the free Khintchine inequality, first proved by M. Bożejko in [B]. The relationship between the classical Khintchine inequality (more precisely its dual version ${ }^{1}$ ) and the free version is the following. Instead of linear combinations of Rademacher functions, we consider functions supported on a subset (of discrete groups) satisfying a certain condition of Leinert (Definition 1.1). In the free case, similar to the classical one, $L_{2 p}$-norms are estimated by $L_{2}$-norms (see Theorem 1.8 below):

$$
\|f\|_{L_{2 p}} \leq C_{p}^{\frac{1}{2 p}}\|f\|_{L_{2}} .
$$

The constants $C_{p}=\left(\begin{array}{c}2 p \\ p\end{array}\right) \frac{1}{p+1}$ (Catalan numbers) are different from the classical ones. The main idea of our proof of the free Khintchine inequality is based on a simple connection (given in Definition 1.3) between the condition of Leinert and

Received by the editors September 23, 1996 and, in revised form, September 3, 1997.

1991 Mathematics Subject Classification. Primary 43A30; Secondary 43A65.

Key words and phrases. Convolution operator, free group, Leinert's set, Khintchine inequality.

This paper is part of the author's Master Thesis under Prof. M. Bożejko, supported by KBN grant 2P03A05108.

${ }^{1}$ We identify the interval $[0,1]$ with the Lebesgue measure, and the group product $\prod_{i \in N} Z_{2}$ with the Haar measure. 
non-crossing pairings. We show the way in which the products (of free generators and its inverses) reducing to identity induce the non-crossing pairings: pairings arise as the elementary deletions between generators and inverses (for more about the connection between Leinert's condition and free generators see Theorem IIIC in $[\mathrm{AO}])$. To give an example: let $a, b$ be free generators; then the product $a b b^{-1} a^{-1}$ is related to only one non-crossing pairing $\{\{1,4\},\{2,3\}\}$, but the product $b a^{-1} a a^{-1} a b^{-1}$ is related to two non-crossing pairings $\{\{1,6\},\{2,3\},\{4,5\}\}$ and $\{\{1,6\},\{2,5\},\{3,4\}\}$. The occurrence of the constants $C_{p}$ in the above inequality has a natural explanation in this proof: the number of non-crossing pairings of totally ordered sets with even cardinality $2 p$ is equal to $C_{p}$. The constants $C_{p}$ are the best in $\left(K_{p}\right)$. It is possible to view this inequality as a certain case of the freecentral-limit-theorem for concrete variables, namely for self-adjoint operators (the free Rademacher functions) $X_{i}=\lambda\left(g_{i}\right)$, where $g_{i}^{2}=e$ freely generate a group. It is obvious that the $X_{i}$ 's form the free family of operators (with the zero expectations and normalized second moments) with respect to the state $\phi$ given by the formula $\phi(T)=\left\langle T \delta_{e} \mid \delta_{e}\right\rangle$. Since

$\lim _{n \rightarrow \infty}\left\|\frac{1}{\sqrt{n}} \sum_{i=1}^{n} X_{i}\right\|_{L_{2 p}}^{2 p}=\lim _{n \rightarrow \infty} \phi\left(\left[\frac{1}{\sqrt{n}} \sum_{i=1}^{n} X_{i}\right]^{2 p}\right)=C_{p}=\int_{-2}^{2} x^{2 p} \frac{1}{2 \pi} \sqrt{4-x^{2}} d x$,

it follows that the spectral measure of the operators $\frac{1}{\sqrt{n}} \sum_{i=1}^{n} X_{i}$ in the state $\phi$ tends to the Wigner measure $d \nu(x)=\frac{1}{2 \pi} \sqrt{4-x^{2}} d x$. We want to point out that in this way we get the elementary proof (without the $R$-transform of Voiculescu) of a free analog of the De Moivre-Laplace central limit theorem.

The purpose of the second section of our paper is to generalize the Haagerup inequality (Lemma 1.4 in $[\mathrm{H}]$ ) on free groups

$$
\left\|\sum_{\left|w_{i}\right|=k} \alpha_{i} \lambda\left(w_{i}\right)\right\| \leq(k+1)\left(\sum\left|\alpha_{i}\right|^{2}\right)^{\frac{1}{2}}, \quad \alpha_{i} \in \mathbb{C},
$$

into the operator case (i.e. $\alpha_{i} \in B(H)$ ). At the beginning of this section we introduce the partial isometry operators $T_{p, q}$, where $p, q$ are any two elements of a free group $G$ (considered with the fixed family of free generators $g_{1}, \ldots, g_{m}$ ). Operators $T_{p, q}$ arise naturally in the following decompositions of the left translations on free groups:

$$
\lambda(p)=\sum_{i=0}^{k} T_{p_{1} \cdots \cdot p_{i}, p_{i+1} \cdots \cdot p_{k}},
$$

where $p \in G$ has reduced writing $p=\prod_{i=1}^{k} p_{i}$, with $p_{i} \in\left\{g_{1}, \ldots, g_{m}, g_{1}^{-1}, \ldots, g_{m}^{-1}\right\}$. The orthogonality of domains and images (for different $q$ and $p$ respectively) of $T_{p, q}$ 's and the decompositions (D) are used to convert the estimates about norms of linear combinations of $\lambda(p)$ 's into estimates about norms of certain matrices. It gives a sequence $\left(H_{k}\right)$ of inequalities (see Theorem 2.8). For $k=1$ we get the estimate (for operator coefficients $\alpha_{i} \in B(H)$ ) as in Haagerup and Pisier's paper (see Proposition 1.1 in $[\mathrm{HP}]$ ):

$$
\left\|\sum_{\left|p_{i}\right|=1} \alpha_{i} \otimes \lambda\left(p_{i}\right)\right\| \leq 2 \max \left\{\left\|\sum \alpha_{i} \alpha_{i}^{*}\right\|^{\frac{1}{2}},\left\|\sum \alpha_{i}^{*} \alpha_{i}\right\|^{\frac{1}{2}}\right\} .
$$


For $k=2$ on the right hand side, besides two previous norms (which are equal to the operator norm of one row and one column matrix respectively), there is the operator norm of the matrix $\left(\alpha_{i, j}\right)$ with zero entries for $\left|p_{i} p_{j}\right| \neq 2$ :

$$
\begin{aligned}
& \left\|\sum_{\left|p_{i} p_{j}\right|=2} \alpha_{i, j} \otimes \lambda\left(p_{i} p_{j}\right)\right\| \\
& \quad \leq 3 \max \left\{\left\|\sum \alpha_{i, j} \alpha_{i, j}^{*}\right\|^{\frac{1}{2}},\left\|\left(\alpha_{i, j}\right)\right\|,\left\|\sum \alpha_{i, j}^{*} \alpha_{i, j}\right\|^{\frac{1}{2}}\right\} .
\end{aligned}
$$

The case $k=3$ is

$\left(H_{3}\right)$

$$
\begin{aligned}
& \left\|\sum_{\left|p_{i} p_{j} p_{k}\right|=3} \alpha_{i, j, k} \otimes \lambda\left(p_{i} p_{j} p_{k}\right)\right\| \\
& \quad \leq 4 \max \left\{\left\|\sum \alpha_{i, j, k} \alpha_{i, j, k}^{*}\right\|^{\frac{1}{2}},\left\|\left(\alpha_{i,(j, k)}\right)\right\|,\left\|\left(\alpha_{(i, j), k}\right)\right\|,\left\|\sum \alpha_{i, j, k}^{*} \alpha_{i, j, k}\right\|^{\frac{1}{2}}\right\},
\end{aligned}
$$

where the rows and columns of the matrix $\left(\alpha_{i,(j, k)}\right)$ are indexed by words of length one and two respectively (conversely for $\left(\alpha_{(i, j), k}\right)$ ), and similarly as before $\alpha_{i,(j, k)}=$ $0\left(\alpha_{(i, j), k}=0\right)$ if $\left|p_{i} p_{j} p_{k}\right| \neq 3$.

\section{The Free Khintchine IneQuality}

First we recall the definition of Leinert's condition.

Definition 1.1. A subset $A$ of a group $G$ satisfies Leinert's condition if for every natural $n$ and any sequence $\left\{g_{i}\right\}_{i=1}^{2 n}$ of elements of $A$ the relation

$$
\prod_{i=1}^{n} g_{2 i-1} g_{2 i}^{-1}=e
$$

implies that $g_{k}=g_{k+1}$ for some $k<2 n$.

The typical example of such a set is the set of free generators. We will present a certain connection between the condition of Leinert and non-crossing pairings defined below.

Definition 1.2. Let $A$ be a finite subset of the natural numbers and let $V$ be a partition of this subset. We say that $V$ is a non-crossing two-partition of $A$ if two conditions hold:

(i) any class of $V$ has exactly two elements,

(ii) there does not exist in $A$ an increasing sequence $a, b, c, d$ such that $a, c$ forms a class and $b, d$ another class of the partition $V$.

We denote by $N_{2}(A)$ the set of all non-crossing two-partitions of the set $A$, and if $A$ is equal to $\{1, \ldots, 2 n\}$ we will write $N C_{2}(2 n)$ instead of $N C_{2}(\{1, \ldots, 2 n\})$.

Definition 1.3. Let a subset $A$ of a discrete group $G$ satisfy Leinert's condition and let $\left\{x_{i}\right\}_{i=1}^{2 n}$ be a sequence of elements of $A$. Let moreover the equality $\prod_{i=1}^{n} x_{2 i-1} x_{2 i}^{-1}=e$ hold. We say that the sequence $\left\{x_{i}\right\}_{i=1}^{2 n}$ induces a non-crossing two-partition $V$, if for any class $\{i, j\}$ of $V$ the equality $x_{i}=x_{j}$ holds.

Proposition 1.4. If a sequence $\left\{x_{i}\right\}_{i=1}^{2 n}$ satisfies the assumptions of Definition 1.3, then the set of non-crossing two-partitions induced by this sequence is not empty. 
Proof. We obtain a simple proof by induction. For $n=1$ the statement of the proposition is obvious. Let a sequence $\left\{x_{i}\right\}_{i=1}^{2 n+2}$ be given. Because Leinert's condition is satisfied, there exists $k<2 n+2$ such that $x_{k}=x_{k+1}$. By the induction assumption there exists a non-crossing two-partition $V$ of the set $\{1, \ldots, 2 n+$ $2\} \backslash\{k, k+1\}$ such that for any class $\{i, j\}$ of $V$ we have $x_{i}=x_{j}$. It is clear that $\widetilde{V}=V \cup\{k, k+1\}$ is a non-crossing two-partition of the set $\{1, \ldots, 2 n\}$.

Proposition 1.5. Let sequences $\left\{x_{i}\right\}_{i=1}^{2 n}$ and $\left\{y_{i}\right\}_{i=1}^{2 n}$ satisfy the assumptions of Definition 1.3. If $\left\{x_{2 i-1}\right\}_{i=1}^{n}=\left\{y_{2 i-1}\right\}_{i=1}^{n}$ and $\left\{x_{2 i}\right\}_{i=1}^{n} \neq\left\{y_{2 i}\right\}_{i=1}^{n}$, then the sets of non-crossing two-partitions induced by these sequences are disjoint.

Corollary 1.6. Let a subset $A$ satisfy Leinert's condition, let $\left\{x_{i}\right\}_{i=1}^{n}$ be a sequence of elements of $A$ and let $\mathbb{A}$ be a class of such sequences. For any fixed $\left\{x_{i}\right\}_{i=1}^{n} \in \mathbb{A}$ the number of elements $\left\{y_{i}\right\}_{i=1}^{n} \in \mathbb{A}$ which satisfy the condition $\prod_{i=1}^{n} x_{i} y_{i}^{-1}=e$ is less than or equal to the cardinality of $N C_{2}(2 n)$, and if the sequence $\left\{x_{i}\right\}_{i=1}^{n}$ has mutually different elements, the equality holds.

Proof of Proposition 1.5. Let $x_{2 k_{0}} \neq y_{2 k_{0}}$. For any $V$ and $\widetilde{V}$, induced by $\left\{x_{i}\right\}_{i=1}^{2 n}$ and $\left\{y_{i}\right\}_{i=1}^{2 n}$ respectively, if $\left\{2 k_{0}, 2 i-1\right\}$ and $\left\{2 k_{0}, 2 j-1\right\}$ are elements of $V$ and $\widetilde{V}$ respectively, then $i \neq j$, because in this case $x_{2 i-1}=x_{2 k_{0}} \neq y_{2 k_{0}}=y_{2 j-1}$ and $\left\{x_{2 i-1}\right\}_{i=1}^{n}=\left\{y_{2 i-1}\right\}_{i=1}^{n}$.

Remark 1.7. The cardinality of $N C_{2}(2 n)$ is equal to the Catalan number $C_{n}=$ $\left(\begin{array}{c}2 n \\ n\end{array}\right)+\frac{1}{n+1}$.

One can find the proof of this fact in the paper of G. Kreweras (Theorem 4 in $[\mathrm{K}])$.

Now we present another proof of the theorem of M. Bożejko (see [B]), see also the paper $[\mathrm{AO}]$, where the operator norm of the operator $\lambda(f)$ is computed; this norm is equal to $\lim _{p \rightarrow \infty}\|f\|_{L_{2 p}}$.

Theorem 1.8 (the free Khintchine inequality). If the support of a function $f: G$ $\rightarrow \mathbb{C}$ satisfies Leinert's condition, then we have the following inequality:

$$
\|f\|_{L_{2 p}} \leq\left[\left(\begin{array}{c}
2 p \\
p
\end{array}\right) \frac{1}{p+1}\right]^{\frac{1}{2 p}}\|f\|_{l^{2}},
$$

where $p$ is a natural number and the constant $\left(\begin{array}{c}2 p \\ p\end{array}\right) \frac{1}{p+1}$ is the best possible for the function with infinite support.

Proof. We have

$$
\begin{aligned}
& \|f\|_{L_{2 p}}^{2 p}=(f * \tilde{f})^{* p}(e) \\
& \quad=\sum_{\left\{\left\{i_{k}\right\}_{k=1}^{2 p} \in\left\{i_{1}, \ldots, i_{n}\right\}^{2 p}: x_{i_{1}} x_{i_{2}}^{-1} \cdots x_{i_{2 p}-1} x_{i_{2 p}}^{-1}=e\right\}} f\left(x_{i_{1}}\right) \tilde{f}\left(x_{i_{2}}\right) \cdots f\left(x_{i_{2 p-1}}\right) \tilde{f}\left(x_{i_{2 p}}\right) \\
& \quad=\sum_{\left\{x_{i}\right\}_{i=1}^{p} \in(\operatorname{supp} f)^{p}} \sum_{\left\{\left\{y_{i}\right\}_{i=1}^{p} \in(\operatorname{supp} f)^{p}: \prod_{i=1}^{p} x_{i} y_{i}^{-1}=e\right\}} \prod_{x \in \operatorname{supp} f}|f(x)|^{2\left[\#\left(\left\{x_{i}\right\}_{i=1}^{p}\right)^{-1}(x)\right]} .
\end{aligned}
$$

From Corollary 1.6 and Remark 1.7 we obtain

$$
\sum_{\left\{\left\{y_{i}\right\}_{i=1}^{p} \in(\operatorname{supp} f)^{p}: \prod_{i=1}^{p} x_{i} y_{i}^{-1}=e\right\}} 1 \leq\left(\begin{array}{c}
2 p \\
p
\end{array}\right) \frac{1}{p+1},
$$


thus

$$
\begin{aligned}
\|f\|_{L_{2 p}}^{2 p} & \leq\left(\begin{array}{c}
2 p \\
p
\end{array}\right) \frac{1}{p+1} \sum_{\left\{x_{i}\right\}_{i=1}^{p} \in(\operatorname{supp} f)^{p}} \prod_{x \in \operatorname{supp} f}|f(x)|^{2\left[\#\left(\left\{x_{i}\right\}_{i=1}^{p}\right)^{-1}(x)\right]} \\
& =\left(\begin{array}{c}
2 p \\
p
\end{array}\right) \frac{1}{p+1}\|f\|_{l^{2}}^{2 p} .
\end{aligned}
$$

In order to prove that the constant $\left(\begin{array}{c}2 p \\ p\end{array}\right) \frac{1}{p+1}$ is the best possible we consider, similarly as in the paper [B], a sequence, normalized with respect to the $l_{2}$-norm, of functions $f_{n}=\frac{1}{\sqrt{n}} \sum_{i=1}^{n} \delta_{g_{i}}$ with support in some infinite subset $A$ which satisfies Leinert's condition. For these functions we have

$$
\begin{aligned}
\left\|f_{n}\right\|_{L_{2 p}}^{2 p} & =\sum_{g_{i_{1}} g_{i_{2}}^{-1} \cdots g_{i_{2 p-1}} g_{i_{2 p}}^{-1}=e}\left(\frac{1}{\sqrt{n}}\right)^{2 p}=\sum_{A} \sum_{B}\left(\frac{1}{\sqrt{n}}\right)^{2 p} \geq \sum_{\widetilde{A}} \sum_{B}\left(\frac{1}{\sqrt{n}}\right)^{2 p} \\
& =\left(\begin{array}{c}
2 p \\
p
\end{array}\right) \frac{1}{p+1} \sum_{\tilde{A}}\left(\frac{1}{\sqrt{n}}\right)^{2 p} \\
& =\left(\begin{array}{c}
2 p \\
p
\end{array}\right) \frac{1}{p+1}\left(\begin{array}{c}
n \\
p
\end{array}\right) p !\left(\frac{1}{\sqrt{n}}\right)^{2 p}
\end{aligned}
$$

where

$$
\begin{aligned}
& A:=\left\{g_{i_{k}}\right\}_{k=1}^{p} \in\left(\operatorname{supp} f_{n}\right)^{p}, \\
& B:=\left\{\left\{h_{k}\right\}_{k=1}^{p} \in\left(\operatorname{supp} f_{n}\right)^{p}: \prod_{k=1}^{p} g_{i_{k}} h_{k}^{-1}=e\right\}, \\
& \widetilde{A}:=\left\{\left\{g_{i_{k}}\right\}_{k=1}^{p} \in\left(\operatorname{supp} f_{n}\right)^{p}: \#\left\{g_{i_{k}}\right\}_{k=1}^{p}(\{1, \ldots, p\})=p\right\} .
\end{aligned}
$$

So the proof of Theorem 1.8 is finished because $\lim _{n \rightarrow \infty}\left(\begin{array}{l}n \\ p\end{array}\right) p !\left(\frac{1}{\sqrt{n}}\right)^{2 p}=1$.

\section{The OPERATOR-VALUED FUnCTIONS}

In this section $E_{k}(G)$ denotes the subset of words of length $k$ of a free group $G$ (considered with the fixed family of free generators $\left.g_{1}, \ldots, g_{m}\right)$. For $p \in E_{k}(G)$ we denote by $p_{i}$ the $i$ th letter in the reduced word $p$, i.e.

$$
p=p_{1} \ldots p_{k}, \quad \text { where } p_{i} \in\left\{g_{1}, \ldots, g_{m}, g_{1}^{-1}, \ldots, g_{m}^{-1}\right\} .
$$

Notations 2.1. Let $G$ be a free group, $h$ an element of $G$ and $p, q$ elements of $E_{k}(G)$ and $E_{l}(G)$ respectively. Then

(i) $l^{2}(G)_{h}$ denotes the closed span of $\left\{\delta_{h g} \in l^{2}(G): g \in G\right.$ and $\left.|h g|=|h|+|g|\right\}$ in $l^{2}(G)$,

(ii) $P_{h}$ denotes the orthogonal projection of $l^{2}(G)$ onto $l^{2}(G)_{h}$,

(iii) $\lambda(h)$ denotes the operator of left convolution by $\delta_{h}$,

(iv) $T_{p, q}$ denotes the operator of the form

$$
\begin{aligned}
& T_{p, q}=\left(\prod_{i=1}^{k} P_{p_{i}} \lambda\left(p_{i}\right)\right)\left(\prod_{i=1}^{l} \lambda\left(q_{i}\right) P_{q_{i}^{-1}}\right)=P_{p} \lambda(p) \lambda(q) P_{q^{-1}}, \\
& T_{p, e}=\prod_{i=1}^{k} P_{p_{i}} \lambda\left(p_{i}\right)=P_{p} \lambda(p) \\
& T_{e, q}=\prod_{i=1}^{l} \lambda\left(q_{i}\right) P_{q_{i}^{-1}}=\lambda(q) P_{q^{-1}} \\
& T_{e, e}=I d
\end{aligned}
$$




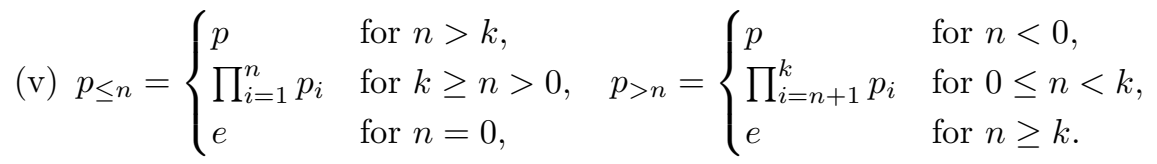

It is very useful that for different $q$ the domains of these operators are orthogonal, and for different $p$ the images are also orthogonal (if we assume that the lengths of $p$ and $q$, respectively, are fixed):

$$
\begin{aligned}
& \text { If } q \neq w \text { and }|q|=|w| \text {, then }\left[\operatorname{Ker}\left(T_{v, w}\right)\right]^{\perp} \perp\left[\operatorname{Ker}\left(T_{p, q}\right)\right]^{\perp}, \\
& \text { If } p \neq v \text { and }|p|=|v| \text {, then } \operatorname{Im}\left(T_{v, w}\right) \perp \operatorname{Im}\left(T_{p, q}\right) \text {. }
\end{aligned}
$$

The operator $T_{p, q}$ has the following properties:

Proposition 2.2. Let $g$ be one of the free generators of the free group $G$ and let $p, p^{\prime}, q, q^{\prime}$ be elements of $E_{k}(G), E_{k^{\prime}}(G), E_{l}(G), E_{l^{\prime}}(G)$ respectively, such that $\left|p p^{\prime}\right|=$ $|p|+\left|p^{\prime}\right|$ and $\left|q q^{\prime}\right|=|q|+\left|q^{\prime}\right|$. Then

(i) $T_{p, e} T_{e, q}=T_{p, q}$,

(ii) $T_{p, e} T_{p^{\prime}, e}=T_{p p^{\prime}, e}$, $T_{e, q} T_{e, q^{\prime}}=T_{e, q q^{\prime}}$

(iii) $\lambda(g)=P_{g} \lambda(g)+\lambda(g) P_{g^{-1}}=T_{g, e}+T_{e, g}$, $\lambda\left(g^{-1}\right)=P_{g^{-1}} \lambda\left(g^{-1}\right)+\lambda\left(g^{-1}\right) P_{g}=T_{g^{-1}, e}+T_{e, g^{-1}}=T_{e, g}^{*}+T_{g, e}^{*}$,

(iv) $\lambda(g) P_{g^{-1}} \lambda\left(g^{-1}\right)=T_{e, g} T_{e, g}^{*}=I d-P_{g}$, $\lambda\left(g^{-1}\right) P_{g} \lambda(g)=T_{g, e}^{*} T_{g, e}=I d-P_{g^{-1}}$,

(v) $T_{e, q} T_{e, q}^{*}=I d-P_{q_{1}}$, $T_{p, e}^{*} T_{p, e}=I d-P_{p_{k}^{-1}}$.

Proof. The statements (i) and (ii) are obvious by the definition. The proof of (iii) is done in the paper [HP] (see proof of Proposition 1.1). Assertion (iv) follows from (iii), because

$$
\lambda(g) P_{g^{-1}} \lambda\left(g^{-1}\right)=\lambda(g)\left[\lambda\left(g^{-1}\right)-\lambda\left(g^{-1}\right) P_{g}\right]=I d-P_{g} .
$$

The statement (v) is a consequence of (iv) and the property (ii).

Corollary 2.3. Let $p, p^{\prime} \in E_{k}(G)$ and $q, q^{\prime} \in E_{l}(G)$. Then

(i) $T_{p, q}^{*} T_{p^{\prime}, q^{\prime}}=\delta_{p, p^{\prime}} T_{e, q}^{*}\left(I d-P_{p_{k}^{-1}}\right) T_{e, q^{\prime}}$,

(ii) $T_{p, q} T_{p^{\prime}, q^{\prime}}^{*}=\delta_{q, q^{\prime}} T_{p, e}\left(I d-P_{q_{1}}\right) T_{p^{\prime}, e}^{*}$.

Proof. We prove only the first equality, the proof of the second is similar. One can observe that if $g, g^{\prime} \in E_{1}(G)$ and $g \neq g^{\prime}$, then

$$
T_{g, e}^{*} T_{g^{\prime}, e}=\lambda\left(g^{-1}\right) P_{g} P_{g^{\prime}} \lambda\left(g^{\prime}\right)=0 .
$$

Hence we obtain

$$
T_{p, e}^{*} T_{p^{\prime}, e}=0, \quad \text { for } p, p^{\prime} \in E_{k}(G) \quad \text { and } \quad p \neq p^{\prime} .
$$

Thus, applying Proposition 2.2 (i) and (v), we get

$$
T_{p, q}^{*} T_{p^{\prime}, q^{\prime}}=T_{e, q}^{*} T_{p, e}^{*} T_{p^{\prime}, e} T_{e, q^{\prime}}= \begin{cases}T_{e, q}^{*}\left(I d-P_{p_{k}^{-1}}\right) T_{e, q^{\prime}} & \text { if } p=p^{\prime}, \\ 0 & \text { if } p \neq p^{\prime} .\end{cases}
$$

Using the above operator $T_{p, q}$ we obtain a decomposition of the left translation operator $\lambda(p)$. This representation is a generalization of the statement (iii) from Proposition 2.2. 
Lemma 2.4. Let $p \in E_{k}(G)$. Then we have the following representation of the operator $\lambda(p)$ :

$$
\lambda(p)=\sum_{i=0}^{k} T_{p_{\leq i}, p_{>i}} .
$$

Proof. We get this equality by applying Proposition 2.2 (iii) to the left translation operator $\lambda(p)=\prod_{i=1}^{k} \lambda\left(p_{i}\right)$.

Now we introduce a matrix representation for a sum of operators $A_{p, q} \otimes T_{p, q} \in$ $B\left(H \otimes_{2} l^{2}(G)\right)$, where $H$ is a Hilbert space. The following notation will be useful:

( $\alpha$ ) for a family $\left\{A_{p_{\leq i}, p_{>i}}\right\}_{p \in E_{k}(G)}$ of bounded operators, the family

$$
\left\{\widetilde{A}_{p, q}\right\}_{(p, q) \in E_{i}(G) \times E_{k-i}(G)}
$$

is defined by the equation

$$
\widetilde{A}_{p, q}= \begin{cases}A_{p, q} & \text { if }|p q|=k, \\ 0 & \text { otherwise }\end{cases}
$$

( $\beta$ ) for a finite family $\left\{A_{p, q}\right\}_{(p, q) \in K \times L}$ of bounded operators acting on a Hilbert space $H$ we denote by $\left(A_{p, q}\right)_{(p, q) \in K \times L}$ the matrix of an operator acting from $\bigoplus_{q \in L} H$ to $\bigoplus_{p \in K} H$.

Lemma 2.5. Let $G$ be a free group with finitely many free generators and let $k$, $i \in \mathbb{N}$ be such that $k \geq i$. Let, moreover, $H$ be a Hilbert space and $\left\{A_{p_{\leq i}, p_{>i}}\right\}_{p \in E_{k}(G)}$ be a family of bounded operators acting on $H$. Then, for the operators

$$
\mathbb{P}_{t}: H \otimes_{2} l^{2}(G) \rightarrow \bigoplus_{w \in E_{t}(G)} H \otimes_{2} l^{2}(G), \quad \text { where } t=k-i, \text { or } t=i,
$$

given by the formulas

$$
\mathbb{P}_{t}(v)=\bigoplus_{w \in E_{t}(G)} P_{w}(v), \quad \text { where } t=k-i, \text { or } t=i,
$$

the following statements hold:

(i) $\left(\widetilde{A}_{p, q} \otimes T_{p, q}\right)_{(p, q) \in E_{i}(G) \times E_{k-i}(G)}=\mathbb{P}_{i} \sum_{p \in E_{k}(G)} A_{p_{\leq i}, p_{>i}}, \otimes T_{p_{\leq i}, p_{>i}} \mathbb{P}_{k-i}^{*}$,

(ii) $\sum_{p \in E_{k}(G)} A_{p_{\leq i}, p_{>i}} \otimes T_{p_{\leq i}, p_{>i}}=\mathbb{P}_{i}^{*}\left(\widetilde{A}_{p, q} \otimes T_{p, q}\right)_{(p, q) \in E_{i}(G) \times E_{k-i}(G)} \mathbb{P}_{k-i}$.

Since both operators $\mathbb{P}_{i}$ and $\mathbb{P}_{k-i}$ have norms equal to one, as a simple consequence we obtain:

Corollary 2.6. Let the assumptions of Lemma 2.5 be satisfied. Then

$$
\begin{aligned}
& \left\|\sum_{p \in E_{k}(G)} A_{p_{\leq i}, p_{>i}} \otimes T_{p_{\leq i}, p_{>i}}\right\|_{B\left(H \otimes_{2} l^{2}(G)\right)} \\
& \quad=\left\|\left(\widetilde{A}_{p, q} \otimes T_{p, q}\right)_{(p, q) \in E_{i}(G) \times E_{k-i}(G)}\right\|_{B(X, Y)},
\end{aligned}
$$

where $X=\bigoplus_{q \in E_{k-i}(G)}\left(H \otimes_{2} l^{2}(G)\right)$ and $Y=\bigoplus_{p \in E_{i}(G)}\left(H \otimes_{2} l^{2}(G)\right)$. 
Proof of Lemma 2.5. The statements of the lemma will become obvious if we observe that the operators $T_{p, q}$ have orthogonal complements of kernels for different $q$ and orthogonal images for different $p$ (with the assumption that the lengths of $p$ and $q$ respectively are fixed); see (2.1).

Indeed, for the first statement (the second one can be shown similarly) we have: if $\delta_{q^{\prime}}$ is not in $l^{2}(G)_{q^{-1}}$ (where $q \in E_{k-i}(G)$ ), then the delta-function $A \otimes \delta_{q^{\prime}}$ (where $A \in H$ ), which lies in the $q$ th orthogonal component (of the orthogonal sum $\left.\bigoplus_{w \in E_{k-i}(G)}\left(H \otimes_{2} l^{2}(G)\right)\right)$, is in the kernels of both operators from statement (i). Now, if $\delta_{q^{\prime}}$ belongs to $l^{2}(G)_{q^{-1}}$ and $A \otimes \delta_{q^{\prime}}$, as a moment ago, belongs to $\left(\bigoplus_{r \in E_{k-i}(G) \backslash\{q\}} H \otimes_{2} l^{2}(G)\right)^{\perp}$ (where orthogonal complement is taken in $\left.\bigoplus_{r \in E_{k-i}(G)}\left(H \otimes_{2} l^{2}(G)\right)\right)$, then for the left-hand side of the equality (i) we have:

$$
\left(\widetilde{A}_{w, l} \otimes T_{w, l}\right)_{(w, l) \in E_{i}(G) \times E_{k-i}(G)} A \otimes \delta_{q^{\prime}}=\bigoplus_{w \in E_{i}(G)} \widetilde{A}_{w, q} A \otimes P_{i+|r|} \delta_{w r},
$$

where $q^{\prime}=q^{-1} r$ and $\left|q^{\prime}\right|=|q|+|r|$, and $P_{k}$ is the projection onto words of length $k$. We obtain the same value for the right-hand side of this equality:

$$
\begin{aligned}
\mathbb{P}_{i} \sum_{p \in E_{k}(G)} A_{p_{\leq i}, p_{>i}} \otimes T_{p_{\leq i}, p_{>i}} \mathbb{P}_{k-i}^{*} A \otimes \delta_{q^{\prime}} \\
=\mathbb{P}_{i} \sum_{p \in E_{k}(G)} A_{p_{\leq i}, p_{>i}} \otimes T_{p_{\leq i}, p>i} \hat{A} \otimes \hat{\delta}_{q^{\prime}} \\
=\mathbb{P}_{i} \sum_{w \in \mathbb{I}} A_{w, q} \hat{A} \otimes P_{i+|r|} \hat{\delta}_{w r} \\
=\bigoplus_{w \in \mathbb{I}} \widetilde{A}_{w, q} A \otimes P_{i+|r|} \delta_{w r} \\
=\bigoplus_{w \in E_{i}(G)} \widetilde{A}_{w, q} A \otimes P_{i+|r|} \delta_{w r},
\end{aligned}
$$

where $\hat{A} \otimes \hat{\delta}_{q^{\prime}}$ means the same function as $A \otimes \delta_{q^{\prime}}$, but in the other space (i.e. in $\left.H \otimes_{2} l^{2}(G)\right), r$ and $P_{k}$ are as above, and $\mathbb{I}$ is the set of these words from $E_{i}(G)$ which multiplied by $q$ are in $E_{k}(G)$.

Lemma 2.7. Let $G$ be a free group with finitely many free generators and let $k, i$ be natural numbers such that $0 \leq i \leq k$. Let, moreover, $H$ be a Hilbert space and $\left\{A_{p_{\leq i}, p_{>i}}\right\}_{p \in E_{k}(G)}$ a family of bounded operators acting on $H$. Then, using the notations

$$
\begin{gathered}
X_{1}=\bigoplus_{q \in E_{k-i}(G)}\left(H \otimes_{2} l^{2}(G)\right), \quad X_{2}=\bigoplus_{p \in E_{i}(G)}\left(H \otimes_{2} l^{2}(G)\right), \\
Y_{1}=\bigoplus_{q \in E_{k-i}(G)} H, \quad Y_{2}=\bigoplus_{q \in E_{i}(G)} H
\end{gathered}
$$

we have

$$
\begin{array}{r}
\left\|\left(\widetilde{A}_{p, q} \otimes T_{p, q}\right)_{(p, q) \in E_{i}(G) \times E_{k-i}(G)}\right\|_{B\left(X_{1}, X_{2}\right)} \\
=\left\|\left(\widetilde{A}_{p, q}\right)_{(p, q) \in E_{i}(G) \times E_{k-i}(G)}\right\|_{B\left(Y_{1}, Y_{2}\right)} .
\end{array}
$$


Proof. In order to prove this equality we show the inequalities on both sides:

( $\geq$ ) Let $v \in Y_{1}$ be a normed vector of the form $v=\bigoplus_{q \in E_{k-i}(G)} v(i)_{q}$. Then $\tilde{v} \in X_{1}$ defined by formula $\tilde{v}=\bigoplus_{q \in E_{k-i}(G)}\left(v(i)_{q} \otimes \delta_{q^{-1}}\right)$ is also normed and satisfies the equation

$$
\left\|\left(\widetilde{A}_{p, q}\right)_{(p, q) \in E_{i}(G) \times E_{k-i}(G)} v\right\|_{Y_{2}}=\left\|\left(\widetilde{A}_{p, q} \otimes T_{p, q}\right)_{(p, q) \in E_{i}(G) \times E_{k-i}(G)} \tilde{v}\right\|_{X_{2}} .
$$

$(\leq)$ In the proof of the opposite inequality we use the following well known facts:

(*) For an operator $T$ acting between Hilbert spaces $H_{1}$ and $H_{2}$ we have

$$
\|T\|_{B\left(H_{1}, H_{2}\right)}^{2}=\left\|T^{*} T\right\|_{B\left(H_{1}\right)}=\left\|T T^{*}\right\|_{B\left(H_{2}\right)},
$$

(**) For a positive operator $T$ (acting on a Hilbert space) which is of the form $T=T_{1}-T_{2}$, where $T_{1}, T_{2}$ are also positive operators, we have $\|T\| \leq\left\|T_{1}\right\|$.

By applying $(*)$ to the operator $\left(\widetilde{A}_{p, q} \otimes T_{p, q}\right)_{(p, q) \in E_{i}(G) \times E_{k-i}(G)}$ we obtain

$$
\begin{aligned}
\|\left(\widetilde{A}_{p, q}\right. & \left.\otimes T_{p, q}\right)_{(p, q) \in E_{i}(G) \times E_{k-i}(G)} \|_{B\left(X_{1}, X_{2}\right)}^{2} \\
& =\left\|\left(\widetilde{A}_{p, q} \otimes T_{p, q}\right)_{(p, q) \in E_{i}(G) \times E_{k-i}(G)}^{*}\left(\widetilde{A}_{p, q} \otimes T_{p, q}\right)_{(p, q) \in E_{i}(G) \times E_{k-i}(G)}\right\|_{B\left(X_{1}\right)} \\
& =\left\|\left(\sum_{p \in E_{i}(G)} \widetilde{A}_{p, q}^{*} \widetilde{A}_{p, r} \otimes T_{p, q}^{*} T_{p, r}\right)_{(q, r) \in E_{k-i}(G) \times E_{k-i}(G)}\right\|_{B\left(X_{1}\right)} \cdot
\end{aligned}
$$

Thus, using Corollary 2.3, we get

$$
\begin{aligned}
\| \widetilde{A}_{p, q} \otimes & \left.T_{p, q}\right)_{(p, q) \in E_{i}(G) \times E_{k-i}(G)} \|_{B\left(X_{1}, X_{2}\right)}^{2} \\
= & \left\|\left(\sum_{p \in E_{i}(G)} \widetilde{A}_{p, q}^{*} \widetilde{A}_{p, r} \otimes\left[T_{e, q}^{*}\left(I d-P_{p_{k}^{-1}}\right) T_{e, r}\right]\right)_{(q, r) \in E_{k-i}(G) \times E_{k-i}(G)}\right\|_{B\left(X_{1}\right)} \\
= & \|\left(\sum_{p \in E_{i}(G)} \widetilde{A}_{p, q}^{*} \widetilde{A}_{p, r} \otimes T_{e, q}^{*} T_{e, r}\right)_{(q, r) \in E_{k-i}(G) \times E_{k-i}(G)} \\
& -\left(\sum_{p \in E_{i}(G)} \widetilde{A}_{p, q}^{*} \widetilde{A}_{p, r} \otimes T_{e, q}^{*} P_{p_{k}^{-1}} T_{e, r}\right)_{(q, r) \in E_{k-i}(G) \times E_{k-i}(G)} \|_{B\left(X_{1}\right)} .
\end{aligned}
$$

From this and $(* *)$ we get the following inequality:

$$
\begin{aligned}
& \left\|\left(\widetilde{A}_{p, q} \otimes T_{p, q}\right)_{(p, q) \in E_{i}(G) \times E_{k-i}(G)}\right\|_{B\left(X_{1}, X_{2}\right)}^{2} \\
& \leq\left\|\left(\sum_{p \in E_{i}(G)} \widetilde{A}_{p, q}^{*} \widetilde{A}_{p, r} \otimes T_{e, q}^{*} T_{e, r}\right)_{(q, r) \in E_{k-i}(G) \times E_{k-i}(G)}\right\|_{B\left(X_{1}\right)} \\
& \quad=\left\|\left(\widetilde{A}_{p, q} \otimes T_{e, q}\right)_{(p, q) \in E_{i}(G) \times E_{k-i}(G)}\right\|_{B\left(X_{1}, X_{2}\right)}^{2} .
\end{aligned}
$$


In a similar way we get

$$
\begin{aligned}
\|\left(\widetilde{A}_{p, q} \otimes\right. & \left.T_{e, q}\right)_{(p, q) \in E_{i}(G) \times E_{k-i}(G)} \|_{B\left(X_{1}, X_{2}\right)}^{2} \\
= & \left\|\left(\widetilde{A}_{p, q} \otimes T_{e, q}\right)_{(p, q) \in E_{i}(G) \times E_{k-i}(G)}\left(\widetilde{A}_{p, q} \otimes T_{e, q}\right)_{(p, q) \in E_{i}(G) \times E_{k-i}(G)}^{*}\right\|_{B\left(X_{2}\right)} \\
= & \|\left(\sum_{q \in E_{k-i}(G)} \widetilde{A}_{p, q} \widetilde{A}_{d, q}^{*} \otimes\left[T_{e, e} T_{e, e}^{*}\right]\right) \\
& -\left(\sum_{q \in E_{k-i}(G)} \widetilde{A}_{p, q} \widetilde{A}_{d, q}^{*} \otimes T_{e, e} P_{q_{1}} T_{e, e}^{*}\right)_{(p, d) \in E_{i}(G) \times E_{i}(G) \times E_{i}(G)} \|_{B\left(X_{2}\right)} \\
\leq & \left\|\left(\widetilde{A}_{p, q} \otimes T_{e, e}\right)_{(p, q) \in E_{i}(G) \times E_{k-i}(G)}\right\|_{B\left(X_{1}, X_{2}\right)}^{2} \\
= & \left\|\left(\widetilde{A}_{p, q}\right)_{(p, q) \in E_{i}(G) \times E_{k-i}(G)}\right\|_{B\left(Y_{1}, Y_{2}\right)}^{2} .
\end{aligned}
$$

So the proof of Lemma 2.7 is finished.

Now we formulate the main theorem of the paper. We should also mention that the result below was known to Uffe Haagerup.

Theorem 2.8. Let $G$ be a free group with finitely many free generators, let $H$ be a Hilbert space and let $f$ be a function supported on $E_{k}(G)$ with values in $B(H)$. Then

(1) $\|\lambda(f)\|_{B\left(H \otimes_{2} l^{2}(G)\right)} \geq \max \left\{\left\|(f(p q))_{(p, q) \in E_{i}(G) \times E_{k-i}(G)}\right\|_{X_{i}}: 0 \leq i \leq k\right\}$,

(2) $\|\lambda(f)\|_{B\left(H \otimes_{2} l^{2}(G)\right)} \leq(k+1) \max \left\{\left\|(f(p q))_{(p, q) \in E_{i}(G) \times E_{k-i}(G)}\right\|_{X_{i}}: 0 \leq i \leq k\right\}$, where $\|\cdot\|_{X_{i}}$ is the operator norm in the space $B\left(\bigoplus_{q \in E_{k-i}(G)} H, \bigoplus_{p \in E_{i}(G)} H\right)$.

Proof. It is clear that $\lambda(f)=\sum_{p \in E_{k}(G)} f(p) \otimes \lambda(p)$. Applying Lemma 2.4 we obtain

$$
\lambda(f)=\sum_{j=0}^{k}\left(\sum_{p \in E_{k}(G)}\left(f(p) \otimes T_{p_{\leq j}, p_{>j}}\right)\right) .
$$

Proof of (1). Let $v(i)$ be a normed vector in $\bigoplus_{q \in E_{k-i}(G)} H$ of the form $v(i)=$ $\bigoplus_{q \in E_{k-i}(G)} v(i)_{q}$. Then the vector $\tilde{v}(i)$ from $H \otimes_{2} l^{2}(G)$ defined by the formula $\tilde{v}(i)=\sum_{q \in E_{k-i}(G)}\left(v(i)_{q} \otimes \delta_{q^{-1}}\right)$ is also normed, and from (*) and (2.1) we have

$$
\begin{aligned}
\|\lambda(f) \tilde{v}(i)\|_{H \otimes_{2} l^{2}(G)}^{2} & =\left\|\sum_{j \geq i} \sum_{p \in E_{k}(G)}\left(f(p) \otimes T_{p_{\leq j}, p_{>j}}\right) \tilde{v}(i)\right\|_{H \otimes_{2} l^{2}(G)}^{2} \\
& =\sum_{j \geq i}\left\|\sum_{p \in E_{k}(G)}\left(f(p) \otimes T_{p_{\leq j}, p_{>j}}\right) \tilde{v}(i)\right\|_{H \otimes_{2} l^{2}(G)}^{2} \\
& \geq\left\|\sum_{p \in E_{k}(G)}\left(f(p) \otimes T_{p_{\leq i}, p>i}\right) \tilde{v}(i)\right\|_{H \otimes_{2} l^{2}(G)}^{2} \\
& =\left\|(f(p q))_{(p, q) \in E_{i}(G) \times E_{k-i}(G)} v(i)\right\|_{\oplus_{p \in E_{i}(G)} H .}^{2}
\end{aligned}
$$


Proof of (2). By $(*)$ we have

$$
\|\lambda(f)\| \leq \sum_{j=0}^{k}\left\|\sum_{p \in E_{k}(G)} f(p) \otimes T_{p_{\leq j}, p>j}\right\|,
$$

thus, it is enough to show that

$$
\left\|\sum_{p \in E_{k}(G)} f(p) \otimes T_{p_{\leq j}, p_{>j}}\right\|_{B\left(H \otimes_{2} l^{2}(G)\right)} \leq\left\|(f(p q))_{(p, q) \in E_{j}(G) \times E_{k-j}(G)}\right\|_{X_{j}} .
$$

But, by Corollary 2.6 and Lemma 2.7, we have the equality since if we denote $A_{p_{\leq j}, p_{>j}}=f\left(p_{\leq j} p_{>j}\right)=f(p)$ for $p \in E_{k}(G)$, then $\widetilde{A}_{p, q}=f(p q)$ for $p \in E_{j}(G)$ and $q \in E_{k-j}(G)$.

Remark. The norms $\|\cdot\|_{X_{i}}$ in the above theorem are similar to the norms $\|\cdot\|_{\{i+1, \ldots, k\}}$ from the paper [HP] (see (0.3) and (0.4) there). The similarity is the following. Let $V$ be a finite set of free generators of a free group $G$ and let $f$ be a function supported on $E_{k}(G)$. We define a family $a_{J}$ of bounded operators on Hilbert space $H$, where $J$ belongs to $\left[V \cup V^{-1}\right]^{k}$, in the following way:

$$
a_{J}=f\left(\prod_{i=1}^{k} J(i)\right), \quad \text { where } \prod_{i=1}^{k} \text { is multiplication in the group } G .
$$

In the above notations we get:

$$
\left\|\left(a_{J}\right)\right\|_{\{i+1, \ldots, k\}}=\left\|(f(p q))_{(p, q) \in E_{i}(G) \times E_{k-i}(G)}\right\|_{X_{i}} .
$$

\section{ACKNOWLEDGMENTS}

I would like to thank Marek Bożejko for introducing me to this subject, and for helpful discussions and suggestions. I also thank the referee for suggestions about the Introduction and other helpful remarks.

\section{REFERENCES}

[AO] C. A. Akemann and Ph. Ostrand, Computing norms in group $C^{*}$-algebras, Amer. J. Math. 98 (1976), 1015-1047. MR 56:1079

[B] M. Bożejko, On $\Lambda(p)$ sets with minimal constant in discrete noncommutative groups, Proc. Amer. Math. Soc. 51(2) (1975), 407-412. MR 52:11481

[H] U. Haagerup, An example of a nonnuculear $C^{*}$-algebra which has the metric approximation property, Invent. Math. 50 (1979), 279-293. MR 80j:46094

[HP] U. Haagerup and G. Pisier, Bounded linear operators between $C^{*}$-algebras, Duke Math. J. 71 (1993), 889-925. MR 94k:46112

[K] G. Kreweras, Sur les partitions non-croisees d'une cycle, Discrete Math. 1 (1972), 333-350. MR 46: 8852

$[\mathrm{Ku}]$ R. A. Kunze, $L_{p}$ Fourier transform on locally compact unimodular groups, Trans. Amer. Math. Soc. 89 (1958), 519-540. MR 20:6668

[M1] M. Leinert, Faltungsoperatoren auf gewissen discreten Gruppen, Studia Math. 52 (1974), 149-158. MR 50:7954

[M2] M. Leinert, Abschatzung von Normen gewisser Matrizen und eine Anwendung, Math. Ann. 240 (1979), 13-19. MR 80j:43004

[P1] G. Pisier, Multipliers and lacunary sets in non-amenable groups, Amer. J. Math. 117 (1992), 337-376. MR 96e:46078

[P2] G. Pisier, The operator Hilbert space OH, complex interpolation and tensor norms, Mem. AMS (1996). MR 97a:46024 
[PP] M. A. Picardello and T. Pytlik, Norms of free operators, Proc. Amer. Math. Soc. 104 (1988), 257-261. MR 90b:47016

[S] R. Speicher, A new example of independence and "white noise", Probab. Th. Rel. Fields $\mathbf{8 4}$ (1990), 141-159. MR 90m:46116

[V1] D. Voiculescu, "Symmetries of some reduced free product $C^{*}$-algebras" in Operator Algebras and Ergodic Theory, Lecture Notes in Math. 1132 (1985), 556-588. MR 87d:46075

[V2] D. Voiculescu, K. Dykema and A. Nica, Free random variables, Amer. Math. Soc. (1992). MR 94c:46133

Institute of Mathematics, University of Wroclaw, Wroclaw pl. Grunwaldzki 2/4, POLAND

E-mail address: buchholz@math.uni.wroc.pl 